\title{
ON A CHARACTERIZATION OF INVARIANT SUBSPACE LATTICES OF WEIGHTED SHIFTS
}

\author{
B. S. YADAV AND S. CHATTERJEE
}

ABSTRACT. The paper concerns itself with the characterization of invariant subspace lattices of weighted shift operators on the Hilbert space $l^{2}$ with suitable conditions on their weights. This characterization is also extended to the case of Banach spaces $l^{p}, 1<p<\infty$.

1. Introduction. Let $l^{2}$ be the Hilbert space of all square-summable complex sequences $x=\left\{x_{0}, x_{1}, x_{2}, \ldots\right\}$ with the norm

$$
\|x\|=\left(\sum_{m=0}^{\infty}\left|x_{m}\right|^{2}\right)^{1 / 2} .
$$

If $\left\{w_{m}\right\}_{m=0}^{\infty}$ is a bounded sequence of nonzero complex numbers, then the operator $T$, defined by

$$
T\left\{x_{0}, x_{1}, x_{2}, \ldots\right\}=\left\{0, w_{0} x_{0}, w_{1} x_{1}, w_{2} x_{2}, \ldots\right\},
$$

is called a unilateral (forward) weighted shift on $l^{2}$ with the weight sequence $\left\{w_{m}\right\}_{m=0}^{\infty}$. We may, and shall, assume without any loss of generality that the weights $w_{m}$ are positive real numbers [2, Problem 2]. By an invariant subspace $M$ of $T$ we shall mean a closed linear manifold of $l^{2}$ such that $T M \subset M$. We shall denote by Lat $T$ the lattice of invariant subspaces of $T$. Various authors have characterized Lat $T$ under suitable conditions on the weight sequence $\left\{w_{m}\right\}_{m=0}^{\infty}$. The following characterization is due to Nikolskir [3]:

If the weight sequence $\left\{w_{m}\right\}_{m=0}^{\infty}$ is monotonically decreasing to zero and belongs to $l^{2}$, then

$$
\text { Lat } T=\left\{\{0\}, M_{1}, M_{2}, \ldots, M_{k}, \ldots, l^{2}\right\} \text {, }
$$

where

$$
M_{k}=\left\{x \in l^{2}: x_{m}=0, m<k\right\} .
$$

The particular case of this result in which $w_{m}=2^{-m}$ was originally obtained by Donoghue [1]; see also [5, p. 66]. The central theme of this paper is to exhibit that Nikolskiî's theorem holds under more general conditions on the weight sequence

Received by the editors July 31, 1979 and, in revised form, May 8, 1981.

1980 Mathematics Subject Classification. Primary 47A15, 47B99.

Key words and phrases. Cyclic sets, Hilbert space, invariant subspaces, weighted shift operators. 
$\left\{w_{m}\right\}_{m=0}^{\infty}$. We shall say that the sequence $\left\{w_{m}\right\}_{m=0}^{\infty}$ is of bounded variation if

$$
\sum_{m=0}^{\infty}\left|w_{m}-w_{m+1}\right|<\infty \text {. }
$$

It is easy to see that if $\left\{w_{m}\right\}_{m=0}^{\infty}$ is monotonically decreasing, then it is of bounded variation, but the converse is not true.

2. We now prove

THEOREM 1. If the weight sequence $\left\{w_{m}\right\}_{m=0}^{\infty}$ is of bounded variation and

$$
\delta=\sup _{m>2, n} \sum_{k=0}^{\infty}\left(\frac{w_{k+m} \cdots w_{k+n}}{w_{m} w_{m+1} \cdots w_{n}}\right)^{2}<\infty,
$$

then Lat $T$ is given by (1).

Proof. Let $\left\{e_{m}\right\}_{m=0}^{\infty}$ be the standard orthonormal basis of $l^{2}$ and let $M$ be an invariant subspace of $T$. Firstly, we proceed to show that if a vector $x=\left\{x_{m}\right\}_{m=0}^{\infty}$, with $x_{0} \neq 0$, is in $M$, then $M=l^{2}$. As

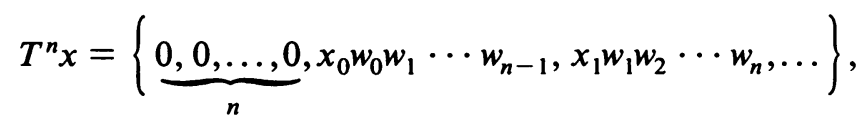

it follows that

$$
\begin{aligned}
\left\|\frac{T^{n} x}{x_{0} w_{0} w_{1} \cdots w_{n-1}}-e_{n}\right\|^{2} & =\sum_{m=0}^{\infty}\left(\frac{w_{m+1} \cdots w_{m+n}}{w_{0} w_{1} \cdots w_{n-1}}\right)^{2}\left|\frac{x_{m+1}}{x_{0}}\right|^{2} \\
& =\frac{w_{n}^{2}}{w_{0}^{2}\left|x_{0}\right|^{2}} \sum_{m=0}^{\infty}\left(\frac{w_{m+1} \cdots w_{m+n}}{w_{1} \cdots w_{n}}\right)^{2}\left|x_{m+1}\right|^{2} \\
& \leqslant \frac{w_{n}^{2}\|x\|^{2}}{w_{0}^{2}\left|x_{0}\right|^{2}} \sum_{m=0}^{\infty}\left(\frac{w_{m+1} \cdots w_{m+n}}{w_{1} \cdots w_{n}}\right)^{2} \\
& =\frac{w_{n}^{2}\|x\|^{2}}{w_{0}^{2} w_{1}^{2}\left|x_{0}\right|^{2}} \sum_{m=0}^{\infty}\left(\frac{w_{m+2} \cdots w_{m+n}}{w_{2} \cdots w_{n}}\right)^{2} w_{m+1}^{2} \\
& =\frac{w_{n}^{2}\|x\|^{2}}{w_{0}^{2} w_{1}^{2}\left|x_{0}\right|^{2}} \sum_{m=0}^{\infty} \sum_{k=0}^{m}\left(\frac{w_{k+2} \cdots w_{k+n}}{w_{2} \cdots w_{n}}\right)^{2}\left(w_{m+1}^{2}-w_{m+2}^{2}\right)
\end{aligned}
$$

(by Abel's transformation [7])

$$
\begin{aligned}
& \leqslant \frac{\delta w_{n}^{2}\|x\|^{2}}{w_{0}^{2} w_{1}^{2}\left|x_{0}\right|^{2}} \sum_{m=0}^{\infty}\left(w_{m+1}^{2}-w_{m+2}^{2}\right) \quad(\text { by }(2)) \\
& \leqslant \frac{\delta w_{n}^{2}\|x\|^{2}}{w_{0}^{2} w_{1}^{2}\left|x_{0}\right|^{2}} \sum_{m=0}^{\infty}\left|w_{m+1}-w_{m+2}\right|\left(w_{m+1}+w_{m+2}\right) \\
& \leqslant \frac{2 \delta \mu w_{n}^{2}\|x\|^{2}}{w_{0}^{2} w_{1}^{2}\left|x_{0}\right|^{2}} \sum_{m=0}^{\infty}\left|w_{m+1}-w_{m+2}\right| \leqslant C w_{n}^{2},
\end{aligned}
$$


where $\mu=\sup _{m}\left\{w_{m}\right\}$ and $C$ is a constant. Since $\left\{e_{n}\right\}_{n=0}^{\infty}$ is an orthonormal basis in $l^{2}$ and $\sum_{n=0}^{\infty} w_{n}^{2}<\infty$, it follows by the Paley-Wiener theorem [6, p. 208] that the system

$$
\left\{\frac{T^{n} x}{x_{0} w_{0} w_{1} \cdots w_{n-1}}\right\}_{n=0}^{\infty}
$$

is a Riesz basis in $l^{2}$, whence we conclude that $M=l^{2}$. Again, if $x_{0}=0$ and $k$ is the least natural number such that $x_{k} \neq 0$, then we can similarly show that

$$
\bigvee_{n=0}^{\infty}\left\{T^{n} x\right\}=M_{k}
$$

Thus we have shown that every cyclic subspace of $T$ is an $M_{k}$. Now our theorem follows by observing that the span of any number of $M_{k}$ 's is again an $M_{k}$.

Our next theorem, which we state without proof, shows that the condition of bounded variation can be dispensed with, provided that condition (2) is replaced by a more stringent condition; even so, our theorem generalizes Nikolskiř's result.

THEOREM 2. If the weight sequence $\left\{w_{m}\right\}_{m=0}^{\infty}$ satisfies the condition

$$
\delta=\sup _{m \geqslant 1, n} \sum_{k=0}^{\infty}\left(\frac{w_{k+m} \cdots w_{k+n}}{w_{m} w_{m+1} \cdots w_{n}}\right)^{2}<\infty,
$$

then Lat $T$ is given by (1).

Now we extend Theorem 2 for the $l^{p}$ spaces, $1<p<\infty$, which, in turn, generalizes Nikolskiǐs main result [3, Theorem 2]. We shall denote by $q$ the Hölder conjugate of $p$, i.e., the number determined by $1 / p+1 / q=1$.

THEOREM 3. Let $T$ be a unilateral (forward) weighted shift on $l^{p}$ with weight sequence $\left\{w_{m}\right\}_{m=0}^{\infty}$ and let

$$
\delta=\sup _{m \geqslant 1, n} \sum_{k=0}^{\infty}\left(\frac{w_{k+m} \cdots w_{k+n}}{w_{m} w_{m+1} \cdots w_{n}}\right)^{q}<\infty .
$$

Then Lat $T$ is given by

$$
\text { Lat } T=\left\{\{0\}, M_{1}, M_{2}, \ldots, M_{k}, \ldots, l^{p}\right\},
$$

where

$$
M_{k}=\left\{x \in l^{p}: x_{m}=0, m<k\right\} .
$$

We shall only sketch the proof of this theorem. Let $M$ be any subspace of $l^{p}$ invariant under $T$. If a vector $x=\left\{x_{m}\right\}_{m=0}^{\infty}, x_{0} \neq 0$, is in $M$, we intend to show that $M=l^{p}$. Let $y=\left\{y_{m}\right\}_{m=0}^{\infty}$ be any element in $l^{q}$ such that $y\left(T^{n} x\right)=0, n=0,1,2, \ldots$ It will suffice to show that $y=0$. We have, for $x_{0}=1$,

$$
y_{n}=\frac{-1}{w_{0} w_{1} \cdots w_{n-1}} \sum_{m=0}^{\infty} w_{m+1} w_{m+2} \cdots w_{m+n} x_{m+1} y_{m+n+1},
$$


and hence, using Hölder's inequality, Abel's transformation and condition (4) in succession, we obtain

$$
\begin{aligned}
\left|y_{n}\right| & \leqslant \frac{w_{n}}{w_{0}}\left(\sum_{m=0}^{\infty}\left|x_{m+1}\right|^{p}\right)^{1 / p}\left(\sum_{m=0}^{\infty}\left(\frac{w_{m+1} \cdots w_{m+n}}{w_{1} w_{2} \cdots w_{n}}\right)^{q}\left|y_{m+n+1}\right|^{q}\right)^{1 / q} \\
& \leqslant \frac{w_{n}}{w_{0}}\|x\|\left(\sum_{m=0}^{\infty} \sum_{k=0}^{m}\left(\frac{w_{k+1} \cdots w_{k+n}}{w_{1} w_{2} \cdots w_{n}}\right)^{q}\left(\left|y_{m+n+1}\right|^{q}-\left|y_{m+n+2}\right|^{q}\right)\right)^{1 / q} \\
& \leqslant \frac{w_{n}}{w_{0}}\|x\| \delta^{1 / q}\left(\sum_{m=0}^{\infty}\left(\left|y_{m+n+1}\right|^{q}-\left|y_{m+n+2}\right|^{q}\right)\right)^{1 / q} \\
& \leqslant 2 \frac{w_{n}}{w_{0}} \delta^{1 / q}\|x\|\|y\| .
\end{aligned}
$$

This inequality is the main step in the proof of Nikolskiî's theorem [3]. By following exactly the line of his argument, we can show that $y=0$, and we are done.

If $x=\left\{x_{m}\right\}_{m=0}^{\infty}$ is a vector in $M$ with $x_{0}=0$, let $k$ be the least positive integer such that $x_{k} \neq 0$. By repeating the argument used above, we obtain $M=M_{k}$. This completes the proof.

3. We now consider the Hilbert space $l^{2}\left(\mathbf{C}^{k}\right), k \geqslant 1$, of norm-square-summable sequences of vectors of the $k$-dimensional unitary spaces $\mathbf{C}^{k}$. Thus $l^{2}\left(\mathbf{C}^{k}\right)$ consists of sequences

$$
x=\left\{x_{m}\right\}_{m=0}^{\infty}, \quad x_{m} \in \mathbf{C}^{k},
$$

such that

$$
\sum_{m=0}^{\infty}\left\|x_{m}\right\|_{*}^{2}<\infty
$$

where $\left\|x_{m}\right\|_{*}$ is the norm of $x_{m}$ in $\mathbf{C}^{k}$, and

$$
\|x\|=\left(\sum_{m=0}^{\infty}\left\|x_{m}\right\|_{*}^{2}\right)^{1 / 2} .
$$

We shall say that a nonempty subset $S$ of $l^{2}\left(\mathbf{C}^{k}\right)$ is a cyclic set of an operator $T$ on $l^{2}\left(\mathbf{C}^{k}\right)$ if

$$
\bigvee_{n=0}^{\infty}\left\{T^{n} x: x \in S\right\}=l^{2}\left(\mathbf{C}^{k}\right)
$$

The following theorem generalises a result due to Nikolskii [4, Lemma 1].

THEOREM 4. Let $T$ be a unilateral weighted shift on $l^{2}\left(C^{k}\right)$ with weight sequence $\left\{w_{m}\right\}_{m=0}^{\infty}$ such that $\left\{w_{m}\right\}_{m=0}^{\infty}$ is of bounded variation and

$$
\delta=\sup _{n} \sum_{k=0}^{\infty}\left(\frac{w_{k+2} \cdots w_{k+n}}{w_{2} \cdots w_{n}}\right)^{2}<\infty .
$$

Then any set of $k$-vectors in $l^{2}\left(\mathbf{C}^{k}\right)$, such that their first coordinates form a basis of $\mathbf{C}^{k}$, is a cyclic set of $T$. 
Proof. Let $x^{(i)}=\left\{x_{m}^{(i)}\right\}_{m=0}^{\infty}, i=1,2, \ldots, k$, be $k$ elements of $l^{2}\left(\mathbf{C}^{k}\right)$ such that $\left\{x_{0}^{(1)}, x_{0}^{(2)}, \ldots, x_{0}^{(k)}\right\}$ is a basis in $\mathbf{C}^{k}$. We assume without any loss of generality that $\left\{x_{0}^{(1)}, x_{0}^{(2)}, \ldots, x_{0}^{(k)}\right\}$ is an orthonormal basis in $\mathbf{C}^{k}$. Then

$$
T^{n} x^{(i)}=\{\underbrace{0,0, \ldots, 0}_{n}, w_{n-1} \cdots w_{1} w_{0} x_{0}^{(i)}, w_{n} \cdots w_{2} w_{1} x_{1}^{(i)}, \ldots\} .
$$

For each $z \in \mathbf{C}^{k}$, let $e_{n}(z)$ denote the element of $l^{2}\left(\mathbf{C}^{k}\right)$ having $z$ in the $n$th place and 0 elsewhere. Now observing that

$$
\left\|x_{m+1}^{(i)}\right\|_{*} \leqslant\left\|x^{(i)}\right\|
$$

and, as in the proof of Theorem 1,

$$
\sum_{m=0}^{\infty}\left(\frac{w_{m+1} \cdots w_{m+n}}{w_{0} w_{1} \cdots w_{n-1}}\right)^{2} \leqslant C w_{n}^{2}
$$

we have

$$
\begin{aligned}
\left\|\frac{T^{n} x^{(i)}}{w_{0} w_{1} \cdots w_{n-1}}-e_{n}\left(x_{0}^{(i)}\right)\right\|^{2} & =\sum_{m=0}^{\infty}\left(\frac{w_{m+1} \cdots w_{m+n}}{w_{0} w_{1} \cdots w_{n-1}}\right)^{2}\left\|x_{m+1}^{(i)}\right\|_{*}^{2} \\
& \leqslant C w_{n}^{2}\left\|x^{(i)}\right\|^{2} .
\end{aligned}
$$

Since

$$
\left\{e_{n}\left(x_{0}^{(i)}\right)\right\}_{n \geqslant 0,1 \leqslant i<k}
$$

is an orthonormal basis in $l^{2}\left(\mathbf{C}^{k}\right)$ and

$$
\sum_{\substack{n \geq 0 \\ 1 \leqslant i \leqslant k}} w_{n}^{2}\left\|x^{(i)}\right\|^{2}<\infty
$$

it follows that the system

$$
\left\{\frac{T^{n} x^{(i)}}{w_{0} w_{1} \cdots w_{n-1}}\right\}_{n \geqslant 0,1<i<k}
$$

is a Riesz basis in $l^{2}\left(\mathbf{C}^{k}\right)$, whence we conclude that $\left\{x^{(i)}\right\}_{i=1}^{k}$ is a cyclic set of $T$.

The authors thank the referee, as also the editor, for their suggestions to rewrite the paper in the present form.

\section{REFERENCES}

1. W. F. Donoghue, The lattice of invariant subspaces of a completely continuous quasi-nilpotent transformation, Pacific J. Math. 7 (1957), 1031-1035.

2. P. R. Halmos, Ten problems in Hilbert space, Bull. Amer. Math. Soc. 76 (1970), 887-933.

3. N. K. Nikolskiir, Invariant subspaces of certain completely continuous operators, Vestnik Leningrad Univ. (7) 20 (1965), 68-77. (Russian)

4. _ Invariant subspaces of weighted shift operators, Math. USSR-Sb. 3 (1967), 159-176.

5. H. Radjavi and P. Rosenthal, Invariant subspaces, Springer-Verlag, Berlin and New York, 1973.

6. F. Riesz and B. Sz.-Nagy, Functional analysis, Ungar, New York, 1955.

7. A. Zygmund, Trigonometric series, Vol. I, 2nd ed., Cambridge Univ. Press, New York, 1959.

Department of Mathematics, University OF Delhi, Delhi-110007, INDIA 